\title{
Energy and target material dependence of the neutron yield induced by proton and deuteron bombardment
}

\author{
Paul Zakalek ${ }^{1, *}$, Paul-Emmanuel Doege ${ }^{1}$, Johannes Baggemann ${ }^{1}$,Eric Mauerhofer ${ }^{1}$ and Thomas Brückel ${ }^{1}$ \\ ${ }^{1}$ Jülich Centre for Neutron Science, Forschungszentrum Jülich GmbH, 52425 Jülich, Germany
}

\begin{abstract}
The neutron yield for compact accelerator driven neutron sources depends on the target material, the ion type and its energy. When such sources are operated with low energy proton beams below $30 \mathrm{MeV}$, typical target materials are lithium and beryllium. New developments indicate that higher energies or a deuteron beam might be useful to increase the neutron yield at constant accelerator power. Here we present the total neutron yield analytically calculated for protons and deuterons at energies up to $100 \mathrm{MeV}$ for various target materials. The total neutron yield depends on the involved cross sections and the stopping power of the target material. This study shows that for energies lower than $30 \mathrm{MeV}$ light target materials with a deuteron beam are preferable whereas for energies above $30 \mathrm{MeV}$ heavy target materials show a high neutron yield with little difference for a proton or deuteron beam.
\end{abstract}

\section{Introduction}

Today, neutron scattering and neutron analytical methods e.g. prompt gamma activation analysis or imaging, are mostly operated at fission or spallation neutron sources. Compact Accelerator-driven Neutron Sources (CANS) operating at low energies are an attractive and costeffective alternative to spallation and fission sources. Several CANS are operated worldwide like the LENS facility in USA [1], the SARAF facility in Israel [2] or the established CANS network in Japan [3]. The recent shutdown of several research reactors in Europe leads to a corresponding reduction in neutron beam days [4]. Projects such as SONATE [5] in France and the HighBrilliance neutron Source project (HBS) $[6,7]$ in Germany work on the development of high-power CANS to counter act the reduction in beam days.

The neutron production at a CANS is based on $(\mathrm{p}, \mathrm{n})$ or $(d, n)$ reactions in a suitable target material initiated by impinging ions with energies in the $\mathrm{MeV}$ range. The energy is below the threshold of spallation reactions $(<100 \mathrm{MeV})$. The neutron yield depends on the ion type (proton, deuteron, etc.), the ion energy and the target material. As all existing CANS are mostly operated with a proton beam at similar energies $(3-13 \mathrm{MeV})$, they use either a beryllium or a lithium target, in order to maximize the neutron yield at these energies $[8,9,10]$.

The development of a high-power CANS with an average deposited power of up to $100 \mathrm{~kW}$ in the target and a peak power up to $10 \mathrm{MW}$ can either be realized by increasing the current or the ion energy. As the ion current is limited to $100-200 \mathrm{~mA}$ with accelerator technologies currently existing, an increase in energy is inevitable. This leads to other target materials than beryllium or lithium for an optimized neutron yield at ion energies above $30 \mathrm{MeV}$. In the following we will present a systematic study of the neutron yield depending on the target material and the energy of a proton or deuteron beam up to $100 \mathrm{MeV}$.

\section{Neutron yield calculations}

Neutrons are produced at a CANS by ions in the $\mathrm{MeV}$ range impinging onto a suitable target i.e. production of many neutrons, high radiation resistance and good thermo-mechanical properties. When the energy of the ions is above the threshold for the $(\mathrm{p}, \mathrm{n})$ or $(\mathrm{d}, \mathrm{n})$ reaction, neutrons are produced. As the ions are decelerated when traveling through the target material, their energy decreases and the corresponding cross section for neutron production changes. The neutron yield for a specific target material therefore depends on the neutron production cross section and the stopping power of the target material.

\subsection{Theory}

The probability $\omega(E)$ that a single ion of energy $E$ undergoes a nuclear reaction with $N_{T}$ target atoms into a reaction channel with the cross section $\sigma(E)$ is given by

$$
\omega(E)=\frac{\sigma(E) N_{T}}{A}
$$

with $A$ as the illuminated target surface. With the Avogadro constant $N_{A}$, the molar mass $M$ and the density $\rho$, the number of target atoms inside a volume $V$ represented by the area $A$ and the thickness $x$ is

\footnotetext{
$\overline{{ }^{*} \text { Corresponding author: p.zakalek@fz-juelich.de }}$
} 


$$
N_{T}=\frac{N_{A}}{M} \rho V=\frac{N_{A}}{M} \rho A x .
$$

As the ions lose energy while traveling through the target, the probability density for a reaction can be written as

$$
\frac{d \omega}{d x}(E)=\frac{N_{A}}{M} \rho \sigma(E)
$$

The formula above must be integrated over the stopping range $R$ or the total loss of projectile energy to get the probability of the reaction

$$
\omega(E \rightarrow 0)=\int_{0}^{R} \frac{N_{A}}{M} \rho \sigma(E) d x .
$$

The cross section for neutron production for a specific ion type at an energy $E$ and a given target element are given in the TENDL data library [11], the ENDF data library [12] or can be calculated using the TALYS nuclear code [13]. The corresponding penetration range for a given energy interval $d E$ can be calculated using the SRIM 2013 toolkit [14]. A python program to calculate the neutron yield was written and uploaded to a GitHub server [15]. The following analysis was done using the TALYS nuclear code using the default parameters as cross sections for all elements can be calculated. The neutron yield was calculated for the natural abundance and a full stop of the ions inside the target material.

\subsection{Neutron production cross section}

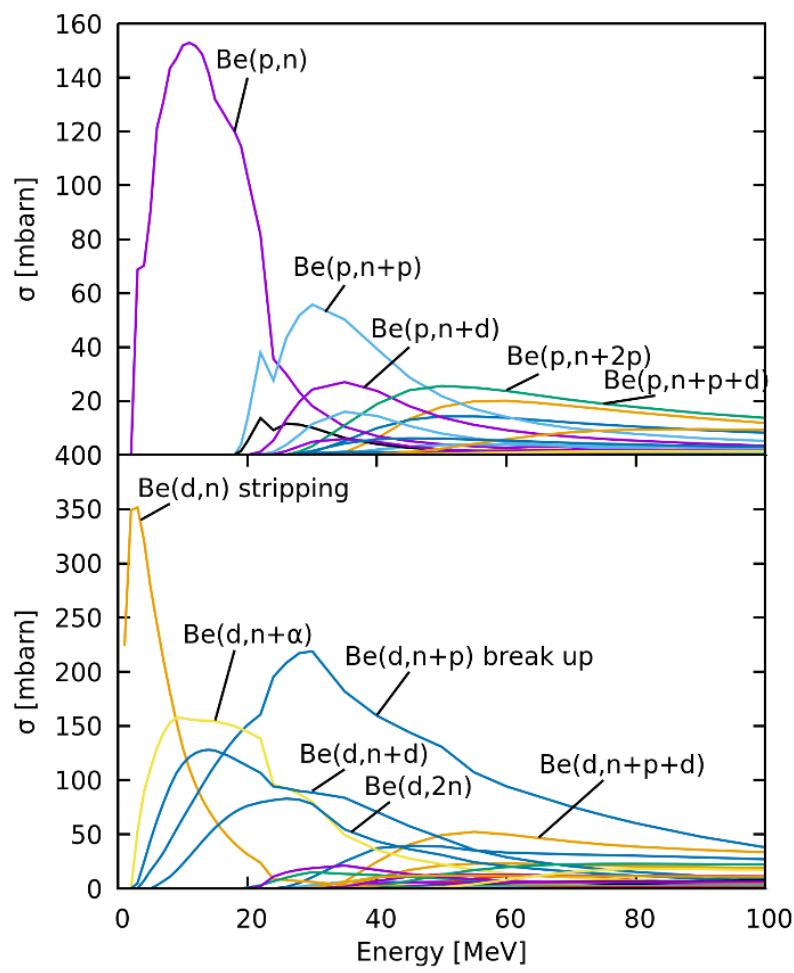

Figure 1: Cross sections for $(p, n)$ reactions (top) and $(d, n)$ reactions (bottom) in beryllium according to the TALYS nuclear code [13].
For a $(\mathrm{p}, \mathrm{n})$ or $(\mathrm{d}, \mathrm{n})$ reaction, the ions need to approach the target atoms close enough. Therefore, they need to overcome the Coulomb barrier. As the target is at rest, the ions need to be accelerated above a specific threshold energy which is depending on the atoms involved. The resulting $(\mathrm{p}, \mathrm{n})$ or $(\mathrm{d}, \mathrm{n})$ reactions depend on the nuclear structure of both atoms, is stochastic in nature and is therefore described by a cross section representing its probability.

In Figure 1 the cross sections for the $(\mathrm{p}, \mathrm{n})$ and $(d, n)$ reactions in a beryllium target are presented. The cross sections for the ${ }^{9} \mathrm{Be}(\mathrm{d}, \mathrm{n})$ reaction are generally larger than the cross sections for the ${ }^{9} \mathrm{Be}(\mathrm{p}, \mathrm{n})$ reactions. This is due to the additional neutron of the deuteron and the possible stripping reaction. In addition, the stripping reaction for deuterons sets in and peaks at low particle energies. The total neutron production cross section for a deuteron beam is in general for all target elements larger than the total neutron production cross section for a proton beam. But it is also necessary to consider the stopping range and therefore the stopping power according to equation 4 .

\subsection{Stopping power and range}

When particles travel through matter, they lose energy due to inelastic scattering. This is described by the linear stopping power

$$
S(E)=-d E / d x,
$$

where $d E$ is the energy loss at initial energy $E$ in the path length $d x$. This energy loss is due to two processes. First, the electronic stopping power due to inelastic collisions of the ion with the electrons of the target and an ionization of the target atoms. This process is described well with the Bethe-Bloch formula. Second, the nuclear stopping power due to a repulsive Coulomb interaction with the target nuclei. This process can be described with a Coulomb interaction potential

$$
V(r)=\frac{Z_{1} Z_{2} e^{2}}{e \pi \varepsilon_{0} r} \Phi(r / a),
$$

with the atomic numbers $Z_{1}$ and $Z_{2}$, the electron charge $e$, the radius $r$, the screening length $a$ and a screening function $\Phi(r / a)$ with the reduced radius $r / a$. A commonly used semi-empirical screening function also used in SRIM was proposed by Ziegler, Littmark and Biersack [16]. Figure 2 shows the electronic and nuclear stopping powers for typically used target materials for protons and deuterons.

As can be seen from Figure 3, the electronic stopping power is orders of magnitude above the nuclear stopping power for light ions like proton and deuteron. Additionally, it is evident that the stopping power for deuterons is higher than for protons resulting in a lower penetration range for the same energy. The stopping range can be determined from the stopping power with

$$
R=\int_{0}^{E} \frac{1}{S(E)} d E
$$




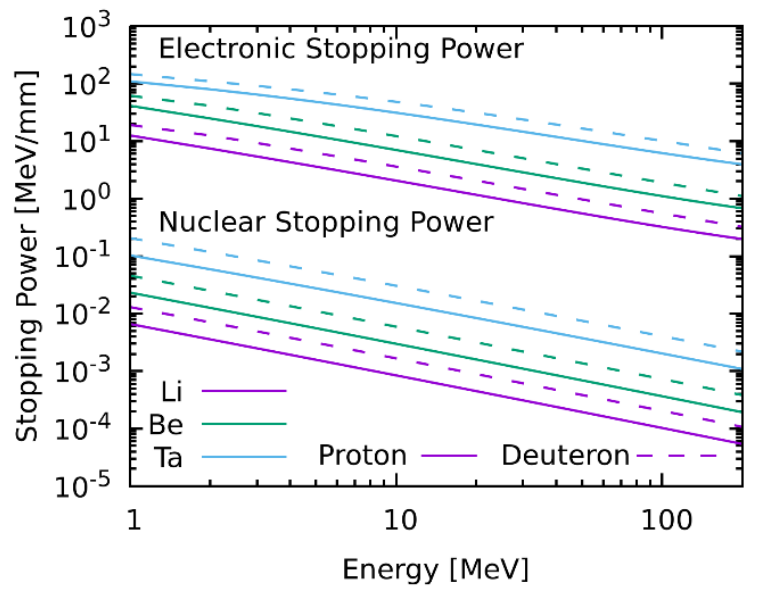

Figure 2: Stopping power, i.e. energy loss per path length traveled for protons and deuterons in $\mathrm{Li}, \mathrm{Be}$ and Ta. The stopping power was calculated using SRIM 2013 [14].

Stopping ranges for typically used target materials and energies for protons and deuterons are presented in Table 1. As mentioned, the probability for a neutronproducing reaction scales linearly with the stopping range. Because of this, the larger cross sections for deuterons in comparison to protons are partly compensated with the larger stopping range for protons.

Table 1. Stopping range for protons and deuterons for various target materials and energies calculated with SRIM 2013 [14].

\begin{tabular}{|c|c|c|c|}
\hline & $\begin{array}{c}\text { Energy } \\
{[\mathrm{MeV}]}\end{array}$ & $\begin{array}{c}\text { Proton } \\
\text { stopping } \\
\text { range } \\
{[\mathrm{mm}]}\end{array}$ & $\begin{array}{c}\text { Deuteron } \\
\text { stopping } \\
\text { range } \\
{[\mathrm{mm}]}\end{array}$ \\
\hline $\mathrm{Li}$ & 5 & 0.91 & 0.53 \\
\hline $\mathrm{Be}$ & 10 & 0.86 & 0.50 \\
\hline $\mathrm{Be}$ & 30 & 6.00 & 3.42 \\
\hline $\mathrm{Ta}$ & 50 & 3.02 & 1.87 \\
\hline $\mathrm{Ta}$ & 70 & 5.34 & 3.28 \\
\hline
\end{tabular}

\subsection{Limitations}

The analysis of the neutron yield relies on the quality of the used cross sections calculated with the TALYS nuclear code [13] or the used database $[11,12]$ and the stopping power calculated with the SRIM toolkit [14].

The electronic stopping power which is well described by the Bethe-Bloch equation is validated well and produces reliable results. The nuclear stopping power calculated with the Ziegler-Biersack-Littmark (ZBL) universal screening potential inside the SRIM toolkit shows deviations for various ions [17]. As the nuclear stopping power is about three orders of magnitude smaller than the electronic stopping power, the stopping powers calculated are still quite reliable.

The relevant cross sections were calculated using the TALYS nuclear code which uses nuclear models to calculate the relevant cross sections. Although the code is tested against experimental data, the quality of the calculated cross sections depends on the isotopes and produces inaccurate cross sections for light elements. Therefore, it is recommended to use specific cross sections from the ENDF/B-VIII database library [12]. The errors for cross sections can be estimated to a factor of three when comparing the calculated cross sections to experimental validations e.g. for beryllium [18]. However, as we are mainly interested in the general trends as function of beam energy and target material, the use of the TALYS nuclear code seems justified.

\section{Results}

Using the method presented, the neutron yield was calculated for protons and deuterons impinging on various elements in the naturally occurring isotopic composition. The neutron yield for the $(\mathrm{p}, \mathrm{n})$ and $(\mathrm{d}, \mathrm{n})$ reactions are presented in Figure 3 and Figure 4, respectively.

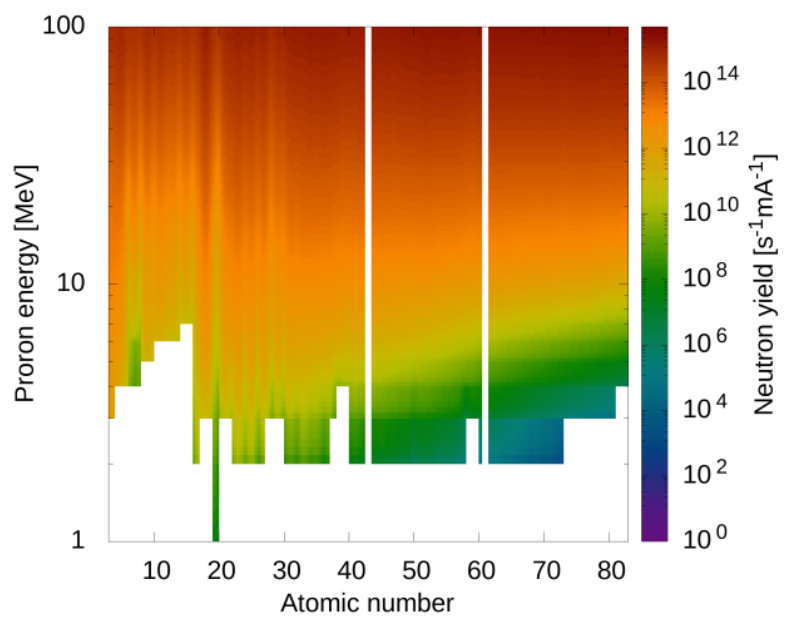

Figure 3: Proton induced neutron yield for various target materials determined from the cross sections calculated with the TALYS nuclear code [13] and the stopping powers calculated with the SRIM 2013 toolkit [14].

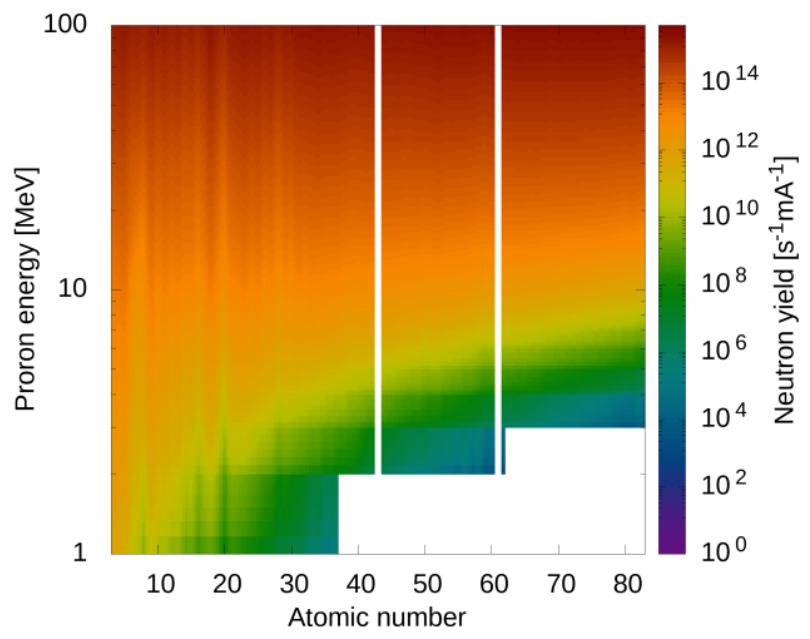

Figure 4: Deuteron induced neutron yield for various target materials determined from the cross sections calculated with the TALYS nuclear code [13] and the stopping powers calculated with the SRIM 2013 toolkit [14]. 
The colour code in the images shows the neutron yield as a function of the ion energy and the target element represented by the atomic number. The elements with the atomic numbers $43(\mathrm{Tc})$ and $61(\mathrm{Pm})$ have no stable isotope and have therefore not been investigated. The $(p, n)$ reaction is endothermic for all elements as the protons need to pass the Coulomb barrier to engage in the reaction and to remove a neutron from the target nuclei, whereas for deuterons, this reaction is exothermic for lighter elements and becomes endothermic for heavier elements due to the stripping reaction channel where the deuteron can easily lose its neutron.

The neutron output increases with increasing energy for protons as well as for deuterons. At energies below 10 $\mathrm{MeV}$, low $\mathrm{Z}$-materials like lithium $(\mathrm{Z}=3)$, beryllium $(\mathrm{Z}=4)$ or argon $(Z=18)$ produce a high number of neutrons for proton induced reactions. Argon is not used as a target material due to the gases state but lithium and beryllium are commonly used target materials. All other elements have a lower neutron output. At intermediate energies around $10 \mathrm{MeV}$, the elements with atomic numbers between 20 and 40 form a transition region in terms of total neutron yield. In this range vanadium $(Z=23)$ shows a high neutron yield and might be an interesting target material. Above $\sim 30 \mathrm{MeV}$ energy, the heavy elements exhibit a high neutron yield without much variation from element to element.

The neutron yield produced by impinging deuterons shows a similar behaviour as for the protons. Below an energy of $30 \mathrm{MeV}$, beryllium has the highest neutron yield. Above this energy, the heavy elements show a similar neutron yield which then exceeds the neutron yield of the light elements.

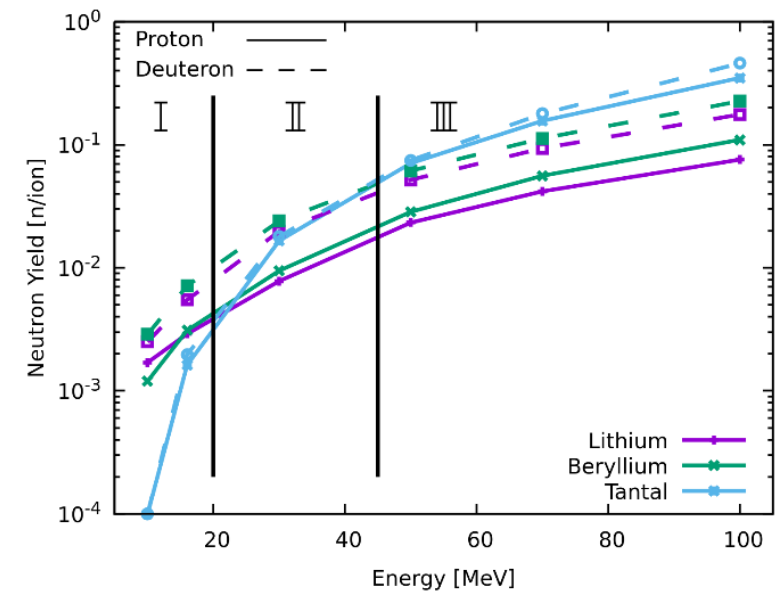

Figure 5: Neutron yield for $\mathrm{Li}, \mathrm{Be}$, and Ta as a function of the primary ion energy. The energy can be seperated into three regions, I, II, and III showing a different behaviour for the neutron yield.

Figure 5 shows the neutron yield induced by proton or deuteron for selected target materials as a function of their energy. For the low energy region I, below $20 \mathrm{MeV}$, the neutron yield is dominated by light elements especially lithium and beryllium. In the transition region II, heavy and light elements show similar neutron yields. The high energy region III is dominated by heavy elements with respect to the neutron yield. As the difference in neutron yield for the heavy elements is not significant, the choice of the material in the high energy region should be taken considering other factors like lifetime, blistering, machinability, etc. A stack of several materials e.g. heavy and light with adopted layer thickness will show a higher neutron yield than a single elemental target, especially in region II.

Figure 6 shows the ratio of the neutron yield for deuterons and protons for various elements. This ratio shows a dependency on the target material as well as on the primary ion energy. For light elements the gain factor when using deuterons is very large but decreases with increasing energy whereas for heavy elements it is lower and rises with the ion energy. The large changes between different target materials for low atomic numbers is most likely due to the inaccurate cross sections calculated with the TALYS nuclear code for light elements.

It can still be deduced that deuterons are preferred at low ion energies or light target elements whereas protons are preferred for high ion energies or heavy target elements.

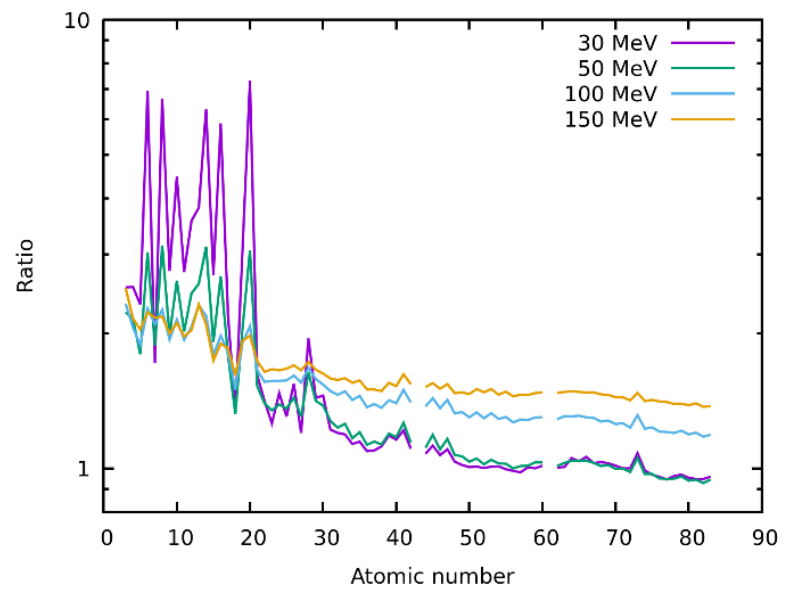

Figure 6: Neutron yield for protons and deuterons as a function of the target element expressed as the deuteron/proton neutron yield ratio.

\section{Discussion}

The analysis of the neutron yield shows a complex behaviour depending on the primary ion type, the energy and the target material.

In general, the choice of the ion type, its energy and the target material depends on the accelerator available. For very low energies $(<5 \mathrm{MeV})$ a lithium target is preferable and is generally used for the neutron production for e.g. boron neutron capture therapy (BCNT) $[9,10]$. The neutron yield can be increased significantly by changing the ion type from protons to deuterons.

For higher energies up to $30 \mathrm{MeV}$, a beryllium target [8, $19]$ is the material of choice and is used at different neutron sources $[1,20]$ or future facilities [5]. The gain factor when changing the ion type from protons to deuterons would still be relevant for such sources but needs careful considerations as the complexity of the 
accelerator increases. The target shows additionally a different thermo-mechanical behaviour when irradiated with a proton or deuteron beam [21].

At high energies, a heavy metal target is the best choice. It offers a large variability regarding the target material and has a high neutron yield. This is the reason why within the HBS project $[6,7]$ a tantalum target was chosen. It has a high neutron yield, can be manufactured well, has good thermo-mechanical properties and has a high blistering threshold [22]. The small gain when using deuterons does not justify the increased accelerator complexity and the larger investment costs. Estimated total neutron yields are presented in Table 2.

Table 2. Calculated total neutron yield for different targets, ion types and energies normalized to the ion current (columns 3,4 ) as well as the source strength normalized to the average power at the target (columns 5,6).

\begin{tabular}{|c|c|c|c|c|c|}
\hline & $\begin{array}{c}\text { Energy } \\
{[\mathrm{MeV}]}\end{array}$ & $\begin{array}{c}\mathbf{p} \\
{\left[\mathrm{s}^{-1} \mathrm{~mA}^{-1}\right]}\end{array}$ & $\begin{array}{c}\mathbf{d} \\
{\left[\mathrm{s}^{-1} \mathrm{~mA}^{-1}\right]}\end{array}$ & $\begin{array}{c}\mathbf{p} \\
{\left[\mathrm{s}^{-1} \mathrm{~kW}^{-1}\right]}\end{array}$ & $\begin{array}{c}\mathbf{d} \\
{\left[\mathrm{s}^{-1} \mathrm{~kW}^{-1}\right]}\end{array}$ \\
\hline $\mathrm{Li}$ & 5 & $3.7 \mathrm{e}+12$ & $5.4 \mathrm{e}+12$ & $7.4 \mathrm{e}+11$ & $1.1 \mathrm{e}+12$ \\
\hline $\mathrm{Be}$ & 10 & $7.7 \mathrm{e}+12$ & $1.8 \mathrm{e}+13$ & $7.7 \mathrm{e}+11$ & $1.8 \mathrm{e}+12$ \\
\hline $\mathrm{Be}$ & 30 & $5.9 \mathrm{e}+13$ & $1.5 \mathrm{e}+14$ & $2.0 \mathrm{e}+12$ & $5.0 \mathrm{e}+12$ \\
\hline $\mathrm{Ta}$ & 50 & $4.4 \mathrm{e}+14$ & $4.7 \mathrm{e}+14$ & $8.8 \mathrm{e}+12$ & $9.3 \mathrm{e}+12$ \\
\hline $\mathrm{Ta}$ & 70 & $9.8 \mathrm{e}+14$ & $1.1 \mathrm{e}+15$ & $1.4 \mathrm{e}+13$ & $1.6 \mathrm{e}+13$ \\
\hline
\end{tabular}

\section{Summary and Outlook}

It was shown that the neutron yield for CANS using $(\mathrm{p}, \mathrm{n})$ and $(\mathrm{d}, \mathrm{n})$ reactions depends on the cross sections for the specific reaction channels and the stopping power / stopping range for the ion type and the target material. A light target material and a deuteron beam are preferable for low energy ion beams, whereas heavy target materials and a proton beam are preferable for high energy ion beams.

Independently of the quality of the cross section calculations, this analysis shows interesting target materials for specific ion types and energies. Therefore, we are planning to perform experiments to determine the total neutron yield for e.g. beryllium, vanadium and tantalum and to compare it with the calculations.

The approach presented in this paper and the algorithm uploaded to GitHub can easily be adapted to calculate the yield of secondary particles e.g. tritium. This will help to screen interesting target materials avoiding time consuming MCNP simulations.

\section{References}

1. C. M. Lavelle, D. V. Baxter, A. Bogdanov, V. P. Derenchuk, H. Kaiser, M. B. Leuschner, M. A. Lone, W. Lozowski, H. Nann, B. v. Przewoski, N. Remmes, T. Rinckel, Y. Shin, W. M. Snow, P. E. Sokol, Nucl. Instrum. Meth. A, 587, 324-341 (2008)
2. I. Mardor, O. Aviv, M. Avrigeanu, D. Berkovits, A. Dahan, T. Dickel, I. Eliyahu, M. Gai, I. GavishSegev, S. Halfon, M. Hass, T. Hirsh, B. Kaiser, D. Kijel, A. Kreisel, Eur. Phys. J. A 54, 91 (2018)

3. Y. Kiyanagi, Eur. Phys. J. Plus 131, 132 (2016)

4. C. Carlile, C. Petrillo, Neutron scattering facilities in Europe: present status and future perspectives, ESFRI Physical Sciences and Engineering Strategy Working Group, June 2016

5. A. Menelle, F. Ott, F. Prunes, B. Homatter, B. Annighöfer, F. Porcher, C. Alba-Simionesco, N. Chauvin, J. Schwindling, A. Letourneau, A. Marchix, N.-H. Tran, arXiv:1612.00237 (2016)

6. U. Rücker, T. Cronert, J. Voigt, J. P. Dabruck, P. -E. Doege, J. Ulrich, R. Nabbi, Y. Beßler, M. Butzek, M. Büscher, C. Lange, M. Klaus, T. Gutberlet, T. Brückel, Phys. J. Plus 131, 19 (2016)

7. P. Zakalek, J. Baggemann, T. Brückel, S. Böhm, T. Cronert, P.-E. Doege, T. Gutberlet, J. Li, E. Mauerhofer, O. Meusel, H. Podlech, M. Rimmler, U. Rücker, M. Schwarz, J. Voigt, International Conference on Heavy Ion Accelerator Technology 14, 117-121 (2019)

8. C.M. Lavelle, D.V. Baxter, A. Bogdanov, V.P. Derenchuk, H. Kaiser, M.B. Leuschner, M.A. Lone, W. Lozowski, H. Nann, B.v. Przewoski, N. Remmes, T. Rinckel, Y. Shin, W.M. Snow, P.E. Sokol, Nucl. Instrum. Meth. A 87, 324-341 (2008)

9. B. Bayanov, V. Belov and S. Taskaev, J. Phys.: Conf. Ser., 41, 460-465 (2006)

10. R. Fujii, Y. Imahori, M. Nakakmura, M. Takada, S. Kamada, T. Hamano, M. Hoshi, H. Sato, J. Itami, Y. Abe, M. Fuse, AIP Conf. Proc., 1509, 162 (2012)

11. A. J. Koning, D. Rochman, J.-Ch. Sublet, N. Dzysiuk, M. Fleming. S. van der Marck, Nucl. Data Sheets, 155, 1-55 (2019)

12. D.A. Brown, et al., Nucl. Data Sheets, 148, 1-142 (2018)

13. A. J. Koning, D. Rochman, Nucl. Data Sheets, 113, 2841-2934 (2012)

14. J. F. Ziegler, A. D. Ziegler, J. P. Biersack, Nucl. Instrum. Meth. B, 268, 1818-1823 (2010)

15. https://iffgit.fz-juelich.de/zakalek/neutron-yield.git

16. J. F. Ziegler, J. P. Biersack, M. D. Ziegler, SRIM: The Stopping and Range of Ions in Matter, SRIM Co., Chester, MD (USA), (2008)

17. H. Paul, AIP Conf. Proc. 1525, 309 (2013)

18. I. Tilquin, P. Froment, M. Cogneau, Th. Delbar, J. Vervier, G. Ryckewaert, Nucl. Instrum. Meth. A, 545, 339 - 343 (2005)

19. P.-E. Doege, P. Zakalek, J. Baggemann, E. Mauerhofer, U. Rücker, T. Cronert, T. Gutberlet, Y. Beßler, J. Wolters, M. Butzek, S. Böhm, R. Nabbi, G. Natour, Th. Brückel, Journal of Neutron Research, 20, 47-54 (2018) 
20. M. Takamura, Y. Ikeda, H. Sunaga, A. Taketani, Y. Otake, H. Suzuki, M. Kumagai, T. Hama, Y. Oba, J. Phys.: Conf. Ser., 734, 032047 (2016)

21. P. Zakalek, P.-E. Doege, J. Baggemann, T. Cronert, Y. Beßler, M. Butzek, J. Wolters, E. Mauerhofer, U. Rücker, T. Gutberlet, G. Natour, Th. Brückel, Physica B, 551, 484 - 487 (2018)

22. V. T. Astrelin, A. V. Burdakov, P. V. Bykov, I. A. Ivanov, A. A. Ivanov, Y. Jongen, S. G. Konstantinov, A. M. Kudryavtsev, K. N. Kuklin, K. I. Mekler, S. V. Polosatkin, V. V. Postupaev, A. F. Rovenskikh, S. L. Sinitskiy, E.R. Zubairov., J. Nucl. Mater., 396, 43 48 (2010) 